\title{
Up to 6-year follow-up after pulmonary vein isolation for persistent/ permanent atrial fibrillation: Importance of sinus node function
}

\author{
Yoshiharu Soga, MD, PhD, ${ }^{a}$ Hitoshi Okabayashi, MD, PhD, ${ }^{\mathrm{b}}$ Yoshio Arai, MD, $\mathrm{PhD},{ }^{\mathrm{c}}$ \\ Takuya Nomoto, MD, PhD, ${ }^{\mathrm{c}}$ Jota Nakano, MD, ${ }^{\mathrm{c}}$ Takehiko Matsuo, $\mathrm{MD},{ }^{\mathrm{c}}$ and Michiya Hanyu, MD, $\mathrm{PhD}^{\mathrm{c}}$
}

Objective: Sinus node dysfunction is commonly associated with atrial fibrillation. There is little information about the long-term results of pulmonary vein isolation in relation to sinus node function. The present study was conducted to investigate whether sinus node dysfunction affects the late outcome of pulmonary vein isolation in patients with persistent/permanent atrial fibrillation.

\begin{abstract}
Methods: Among 76 consecutive patients with persistent/permanent atrial fibrillation who had undergone cutand-sew pulmonary vein isolation, 66 patients without evidence of intra-atrial thrombus by transesophageal echography, and who were able to tolerate cardioversion, were enrolled. Sinus node recovery time after cardioversion was examined intraoperatively. All of the patients underwent valvular surgery concomitantly (mitral in 62).

Results: Sinus node dysfunction was detected in 18 patients. These patients had a significantly lower $\mathrm{f}$ wave voltage in $\mathrm{V}_{1}$ of the electrocardiogram and a larger cardiothoracic ratio than patients with normal sinus node function. Hospital mortality was $3 \%$, and 3 late deaths were observed. Follow-up was conducted for up to 72 months (mean 30 months), with a $100 \%$ complete follow-up rate. There were no significant differences in actuarial survival and freedom from cardiac events between patients with normal and abnormal sinus node function. No thromboembolic events occurred. A significantly higher proportion of patients with normal sinus node function $(82 \%)$ were free of atrial fibrillation at 4 years than patients with sinus node dysfunction $(25 \% ; P<.0001)$.

Conclusions: The atrial fibrillation cure rate after pulmonary vein isolation may be influenced by sinus node function in both the early and late stages. Although further examinations are required, pulmonary vein isolation may be an adequate treatment for persistent/permanent atrial fibrillation in patients with normal sinus node function. (J Thorac Cardiovasc Surg 2011;141:1455-60)
\end{abstract}

Although the Cox maze III procedure has been widely recognized as the most effective surgical treatment for patients with persistent/permanent atrial fibrillation (AF), pulmonary vein isolation (PVI) has recently been increasingly adopted along with the introduction of energy-based techniques. ${ }^{1-4}$ From a surgical viewpoint, PVI is preferable because it requires a smaller lesion set, thus reducing the time required for aortic crossclamping and extracorporeal circulation. However, it is still unknown whether PVI is sufficient for the treatment of persistent/permanent AF. The mechanisms of persistent/permanent AF have been not fully clarified, and the usage of various energy sources may alter the results, as is the case for modifications of

\footnotetext{
From the Department of Cardiovascular Surgery, ${ }^{\mathrm{a}}$ Nara Hospital, Kinki University School of Medicine, Ikoma; Department of Cardiovascular Surgery, ${ }^{\mathrm{b}}$ Iwate Medical University Memorial Heart Center, Morioka; and Department of Cardiovascular Surgery, ${ }^{\mathrm{c}}$ Kokura Memorial Hospital, Kitakyushu, Japan.

Disclosures: Authors have nothing to disclose with regard to commercial support.

Received for publication Sept 9, 2009; revisions received March 8, 2010; accepted for publication April 3, 2010; available ahead of print Sept 13, 2010.

Address for reprints: Yoshiharu Soga, MD, PhD, Assistant Professor, Department of Cardiovascular Surgery, Nara Hospital, Kinki University School of Medicine, 1248-1 Otoda-cho, Ikoma, Nara, 630-0293, Japan (E-mail: sogakin@dd.iij4u.or. jp).

$0022-5223 / \$ 36.00$

Copyright (C) 2011 by The American Association for Thoracic Surgery doi:10.1016/j.jtcvs.2010.04.045
}

the maze procedure. ${ }^{5,6}$ In addition, there are few reports about the late results of classic cut-and-sew PVI.

In the early Cox maze I/II era, a high incidence of pacemaker implantation after the procedure was reported. ${ }^{7,8}$ The surgical technique was therefore modified to avoid incisional damage to the sinus node $(\mathrm{SN})$ artery, and thus the Cox maze III procedure evolved. ${ }^{9}$ However, a proportion of patients still require pacemaker implantation after Cox maze III, and even after PVI, suggesting that some candidates for AF surgery have underlying SN dysfunction.

It is widely known that $\mathrm{SN}$ dysfunction is commonly associated with $\mathrm{AF}$ and that patients with sick sinus syndrome (SSS) frequently have AF. ${ }^{10,11}$ Therefore, we have hypothesized that some patients who are surgical candidates for AF have SN dysfunction and that PVI would be an insufficient treatment for them. Here we report the long-term results of cut-and-sew PVI for up to 6 years, focusing on SN function.

\section{PATIENTS AND METHODS \\ Patients}

Between May 2002 and September 2007, 88 consecutive patients underwent cut-and-sew PVI at a single institution (Kokura Memorial Hospital). Of these patients, 76 had persistent/permanent AF preoperatively. Intraoperative transesophageal echocardiography revealed no evidence of intracardiac thrombus in 66 patients. These 66 were thought to be capable of tolerating direct-current cardioversion and were enrolled in the present 


$$
\begin{aligned}
& \text { Abbreviations and Acronyms } \\
& \text { AF }=\text { atrial fibrillation } \\
& \text { ECG }=\text { electrocardiogram } \\
& \text { PVI }=\text { pulmonary vein isolation } \\
& \text { SN }=\text { sinus node } \\
& \text { SSS }=\text { sick sinus syndrome }
\end{aligned}
$$

study. Written consent was obtained from all patients after a full explanation had been given before the operation. The study was approved by the Institutional Review Board of Kokura Memorial Hospital in compliance with the Declaration of Helsinki.

Immediately after the initiation of cardiopulmonary bypass, cardioversion was performed before aortic crossclamping. If the heart was defibrillated, the overdrive suppression test was used and SN recovery time was assessed. Patients were then divided into 2 groups: (1) a normal SN function group $(\mathrm{n}=48)$, which was defined as SN recovery time within $1 \mathrm{sec}$ ond, and (2) an SN dysfunction group $(\mathrm{n}=18)$, which was defined as SN recovery time of more than 1 second $(n=10)$ or not defibrillated $(n=8) .{ }^{12}$ Preoperative demographics are summarized in Table 1. Patients with SN dysfunction had a significantly lower $f$ wave voltage in $V_{1}$ of the electrocardiogram (ECG) and a larger cardiothoracic ratio on chest x-ray films than patients with normal SN function. Age, left atrial dimension, and left ventricular ejection fraction did not differ between the groups.

\section{Surgical Technique}

After the examination of SN function, the aorta was clamped, and warm blood cardioplegic solution was infused for myocardial protection. A rightsided vertical incision in the left atrium was made and extended to encircle all the pulmonary veins (as a box lesion), leaving 1.5 to $2 \mathrm{~cm}$ near the left pulmonary vein to prevent separating the left atrium completely. Cryoablation was applied to this remnant for 180 seconds. The left atrium was closed with continuous sutures of 4-0 polypropylene. Operative procedures are shown in Table 2. Mitral valve surgery was performed in $94 \%(62 / 66)$ of the patients. Aortic clamping time and cardiopulmonary bypass time did not differ significantly between the groups, irrespective of the concomitant procedures (Table 2).

\section{Postoperative Management and Follow-up}

Both aspirin and warfarin were administrated to patients with valve prostheses (19 patients in the normal SN function group and 11 in the SN dysfunction group) and to patients without valve prostheses but having frequent paroxysmal $\mathrm{AF}$ or sustained $\mathrm{AF}$ postoperatively ( 1 in the normal $\mathrm{SN}$ function group and 5 in the SN dysfunction group). Antiarrhythmic agents were not used prophylactically. Postoperative AF was treated mainly with pilsicainide hydrochloride and with cardioversion in cases of drug-resistant AF during hospitalization.

All information was collected and categorized in accordance with the latest guidelines published in $2007 .{ }^{13}$ Patients were followed up at our outpatient clinic at 1, 3, and 6 months after discharge and then annually, and ECGs were recorded every time. Sixty of 61 survivors were followed up at our outpatient clinic, and the remaining 1 patient, who had been hospitalized for treatment of dementia, was contacted directly by the physician. The rate of complete follow-up was $100 \%$. The cumulative follow-up time was 162.3 patient-years, with a mean observation time of 29.5 months and

\begin{tabular}{|c|c|c|c|}
\hline & $\begin{array}{c}\text { Normal function } \\
\text { group }(n=48)\end{array}$ & $\begin{array}{c}\text { Dysfunction } \\
\text { group }(n=18)\end{array}$ & $\begin{array}{c}P \\
\text { value }\end{array}$ \\
\hline Gender (male/female) & $35 / 13$ & $6 / 12$ & .003 \\
\hline Age (y) & $63.6 \pm 1.7$ & $68.7 \pm 2.4$ & .105 \\
\hline NYHA I & $7(15 \%)$ & $1(6 \%)$ & .317 \\
\hline NYHA III/IV & $12(25 \%)$ & $5(28 \%)$ & .818 \\
\hline History of embolism & $8(17 \%)$ & $4(22 \%)$ & .602 \\
\hline Anticoagulant usage & $39(81 \%)$ & $17(94 \%)$ & .293 \\
\hline Aspirin alone & $10(21 \%)$ & 0 & .031 \\
\hline Warfarin alone & $3(6 \%)$ & $2(11 \%)$ & .539 \\
\hline Aspirin + warfarin & $26(54 \%)$ & $15(83 \%)$ & .045 \\
\hline Antiarrhythmia drug usage & $36(75 \%)$ & $15(83 \%)$ & .472 \\
\hline Digitalis alone & 13 & 9 & \\
\hline Beta-blocker alnoe & 6 & 1 & \\
\hline Verapamil alone & 5 & 1 & \\
\hline Digitalis + beta-blocker & 3 & 2 & \\
\hline $\begin{array}{l}\text { Digitalis + beta-blocker } \\
+ \text { Verapamil }\end{array}$ & 2 & 0 & \\
\hline Digitalis + verapamil & 1 & 1 & \\
\hline Digitalis + pilsicainide & 1 & 0 & \\
\hline Digitalis + pimenol & 1 & 0 & \\
\hline Beta-blocker + Verapamil & 2 & 0 & \\
\hline $\begin{array}{l}\text { Beta-blocker + verapamil } \\
\quad+\text { Pilsicainide }\end{array}$ & 1 & 0 & \\
\hline Verapamil + pilsicainide & 1 & 0 & \\
\hline Amiodarone alone & 0 & 1 & \\
\hline $\mathrm{f}$ Wave voltage in $\mathrm{V}_{1}(\mathrm{mV})$ & $0.14 \pm 0.01$ & $0.07 \pm 0.02$ & .003 \\
\hline CTR $(\%)$ & $55.7 \pm 0.8$ & $61.6 \pm 1.7$ & .005 \\
\hline $\mathrm{LAD}(\mathrm{mm})$ & $50.5 \pm 1.1$ & $53.4 \pm 1.7$ & .167 \\
\hline $\operatorname{LVEF}(\%)$ & $60.9 \pm 1.8$ & $62.3 \pm 1.9$ & .649 \\
\hline Mitral valve disease & $44(92 \%)$ & $18(100 \%)$ & .206 \\
\hline Degenerative MR & $27(56 \%)$ & $12(67 \%)$ & .443 \\
\hline
\end{tabular}
a range of 0.2 to 72 months.

\section{Statistical Analysis}

All values were expressed as mean \pm standard deviation. Continuous variables were compared using the nonparametric Mann-Whitney $U$ test.
TABLE 1. Preoperative patient demographics

NYHA, New York Heart Association; CTR, cardiothoracic ratio; $L A D$, left atrial dimension; $L V E F$, left ventricular ejection fraction; $M R$, mitral regurgitation

Proportions were compared using Fisher's exact test. The event-free survival curves were constructed using the Kaplan-Meier method, and differences between the groups were investigated by log-rank test.

\section{RESULTS}

\section{Early Results}

Operative mortality was $1.5 \%(\mathrm{n}=1)$, and hospital mortality was $3 \%(n=2)$. One patient with poor left ventricular function and chronic renal dysfunction, who had undergone both aortic and mitral valve replacement concomitantly, died of multiple organ failure with systemic inflammatory response syndrome on the sixth postoperative day. Another patient, who had undergone aortic valve replacement using a stentless valve prosthesis, died of mediastinitis on the 48th postoperative day. Both were in the normal SN function group. Nonfatal cerebral infarction occurred in another 2 patients in the normal SN function group during hospitalization; one patient had Paf, and the other had no AF postoperatively. One patient in the $\mathrm{SN}$ dysfunction group underwent pacemaker implantation for SSS before 
TABLE 2. Operative procedure

\begin{tabular}{lccc}
\hline & $\begin{array}{c}\text { Normal function } \\
\text { group }(\mathbf{n}=\mathbf{4 8})\end{array}$ & $\begin{array}{c}\text { Dysfunction group } \\
(\mathbf{n}=\mathbf{1 8})\end{array}$ & $\begin{array}{c}\boldsymbol{P} \\
\text { value }\end{array}$ \\
\hline Mitral valve procedure & 44 & 18 & .206 \\
$\quad$ Repair & 31 & 11 & \\
$\quad$ Replacement & 13 & 7 & \\
Aortic valve/root & 10 & 4 & .902 \\
$\quad$ replacement & & & \\
$\quad$ Isolated & 4 & 0 & \\
$\quad$ Concomitant & 6 & 4 & \\
Coronary artery bypass & 5 & 3 & .537 \\
Tricuspid annuloplasty & 4 & 1 & \\
ASD closure & 1 & 1 & \\
LV volume reduction & 1 & 0 & \\
LAA resection & 1 & 0 & \\
Aortic clamp time (min) & $92.8 \pm 4.6$ & $83.6 \pm 8.3$ & .308 \\
CPB time (min) & $118.5 \pm 4.9$ & $110.1 \pm 10.0$ & .406 \\
\hline
\end{tabular}

$A S D$, Atrial septal defect; $L V$, left ventricular; $L A A$, left atrial appendage; $C P B$, cardiopulmonary bypass.

discharge. All of these early events were included the late event analysis.

Postoperative AF was detected in $19(41 \%)$ of the patients in the normal SN function group and in $17(94 \%)$ of those in the SN dysfunction group during hospitalization, and this difference was significant $(P<.0001)$. Among them, cardioversion was used for drug-resistant $\mathrm{AF}$ in 12 patients of the normal SNF group and 6 patients of the SN dysfunction group, respectively. At the time of discharge, $42(91 \%)$ patients in the normal SN function group and $6(33 \%)$ in the $\mathrm{SN}$ dysfunction group were in sinus rhythm, and this difference was also significant $(P<.0001)$. With regard to use of antiarrhythmic agents, there was no significant intergroup difference; 24 patients in the normal $\mathrm{SN}$ function group and 10 in the SN dysfunction group $(P=.87)$.

\section{Late Results}

There were 3 late deaths ( $1.8 \%$ /patient-year). Two patients in the normal SN function group died of noncardiac causes: liver dysfunction owing to cirrhosis and malignancy, respectively. One patient in the SN dysfunction group, who had been administered warfarin for longstanding $\mathrm{AF}$, died of cerebral hemorrhage.
Five nonfatal cardiac events occurred in the normal SN function group: catheter ablation for atrial tachycardia or paroxysmal $\mathrm{AF}$ in 2, redo mitral valve surgery in 2 (rerepair and replacement, respectively), and percutaneous coronary intervention in 1 . The patient who underwent catheter ablation for paroxysmal AF underwent pacemaker implantation later.

In the SN dysfunction group, 3 cardiac events were observed: implantation of a pacemaker for SSS in 2 and of an implantable cardioverter defibrillator for ventricular tachycardia in 1.

Actuarial freedom from death owing to all causes at 4 years was $85.1 \% \pm 8.7 \%$ in the normal $\mathrm{SN}$ function group and $94.1 \% \pm 5.7 \%$ in the $\mathrm{SN}$ dysfunction group (Figure 1, A). Actuarial freedom from cardiac death at 3 years was $100 \%$ in the normal SN function group and $94.1 \% \pm$ $5.7 \%$ in the SN dysfunction group (Figure 1, B). Late thromboembolic events were not observed in either group.

During the follow-up period, AF was detected in $6(13 \%)$ patients in the normal SN function group and $11(61 \%)$ in the $\mathrm{SN}$ dysfunction group, and this difference was significant $(P<.0001)$. Actuarial freedom from cardiac events at 4 years was $83.3 \% \pm 6.7 \%$ in the normal $\mathrm{SN}$ function group and $77.4 \% \pm 10.0 \%$ in the $\mathrm{SN}$ dysfunction group. There was no significant difference between the groups (Figure 2, A).

Actuarial freedom from $\mathrm{AF}$ at 4 years in the normal SN function group $(82.3 \% \pm 5.7 \%)$ was significantly better than that in the SN dysfunction group $(25.4 \% \pm 12.9 \%)$ $(P<.001$; Figure $2, B)$. At the time of the latest followup, the ECG showed sinus rhythm in $88 \%$ of patients in the normal SN function group, and this was also significantly better than for patients in the $\mathrm{SN}$ dysfunction group $(41 \% ; P<.001)$. Percentages of patients with sinus rhythm confirmed by ECG during each follow-up period are shown in Figure 3. About $90 \%$ of patients in the normal SN function group maintained sinus rhythm, compared with about $40 \%$ of patients in the SN dysfunction group.

Anticoagulation therapy was used in 31 of the patients in the normal SN function group and in 16 of the patients in the $\mathrm{SN}$ dysfunction group $(P=.17)$. Among them, warfarin was used more frequently in the $\mathrm{SN}$ dysfunction group
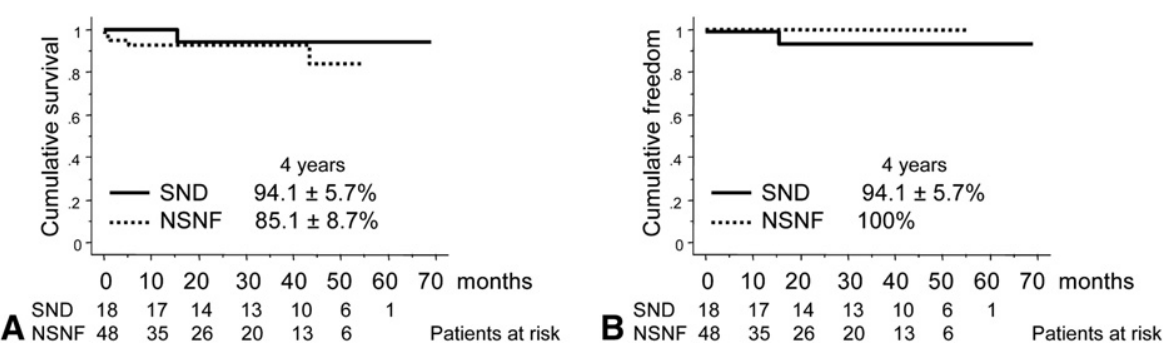

FIGURE 1. A, Actuarial freedom from death owing to all causes. B, Actuarial freedom from cardiac deaths. SND, Sinus node dysfunction; NSNF, normal sinus node function. 

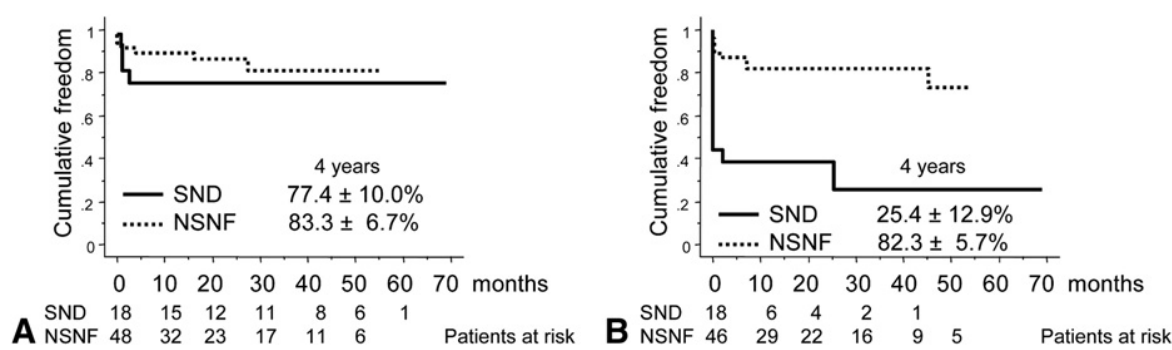

FIGURE 2. A, Actuarial freedom from cardiac events. B, Actuarial freedom from atrial fibrillation. $S N D$, Sinus node dysfunction; $N S N F$, normal sinus node function.

$(\mathrm{n}=16,89 \%)$ than in the normal $\mathrm{SN}$ function group (n $=20,42 \% ; P=.003)$.

\section{DISCUSSION \\ Major Findings}

In the present study, we compared the results of cut-andsew PVI for persistent/permanent AF associated with heart valve disease for up to 6 years between patients with normal and abnormal SN function. The key findings were as follows: (1) Preoperatively, patients with SN dysfunction had a significantly lower $\mathrm{f}$ wave voltage in $\mathrm{V}_{1}$ of the ECG and a larger cardiothoracic ratio on chest $\mathrm{x}$-ray films than patients with normal SN function; (2) at the time of discharge, sinus rhythm was maintained in $42(91 \%)$ of the patients with normal SN function, compared with $6(33 \%)$ of the patients with SN dysfunction $(P<.0001)$; (3) there were no significant differences between the groups in terms of freedom from death and cardiac events; (4) no late thromboembolic events were observed in either of the groups; (5) the rate of freedom from $\mathrm{AF}$ at 3 years in patients with normal SN function $(91 \%)$ was significantly higher than that in patients with SN dysfunction.

\section{Effect of PVI on Persistent/Permanent AF}

The results of PVI seem to be comparable with those of the Cox maze procedure in patients with paroxysmal AF. ${ }^{5}$ However, it is still not clear whether PVI is an adequate treatment for patients with persistent/permanent AF. The lack of

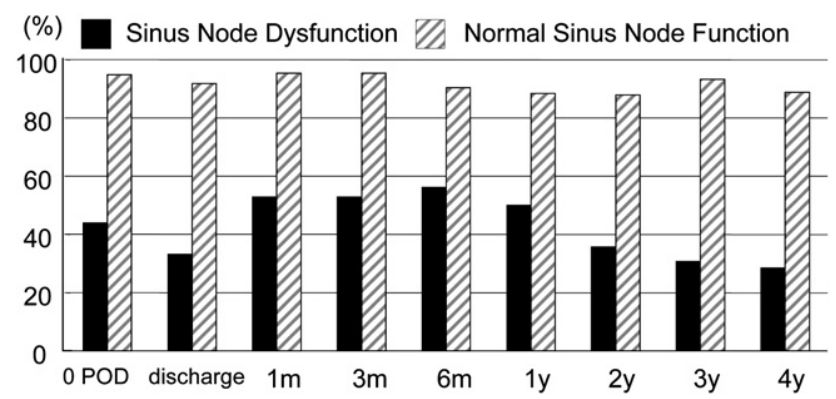

FIGURE 3. Percentage of sinus rhythm confirmed by electrocardiogram during each follow-up period. $P O D$, Postoperative day. long-term results for PVI, especially using the most reliable cut-and-sew technique, may be a major reason.

Kalil and colleagues ${ }^{1}$ have reported satisfactory early results of cut-and-sew PVI in 15 patients with chronic AF and associated mitral valve disease, of whom $87.5 \%$ had achieved sinus rhythm by 1 year after the operation. Sueda and associates ${ }^{2}$ reported the results of PVI with the aid of cryoablation or radiofrequency ablation for up to 4 years in 49 patients with chronic persistent $\mathrm{AF}$ and heart disease, including 41 patients with valvular disease. Of these patients, $71.4 \%$ and $70.2 \%$ were in sinus rhythm at discharge and at the last follow-up, respectively. In a comparative follow-up study of full maze, limited maze, and PVI with the aid of radiofrequency ablation, Melby and associates ${ }^{3}$ reported that $59 \%$ of patients who received PVI were free of AF at the time of follow-up. Another study by Gillinov and colleagues ${ }^{4}$ showed that $66 \%$ of patients were free of AF 18 months after radiofrequency ablation and clearly demonstrated that permanent $\mathrm{AF}$ was a risk factor for AF recurrence. In the present study, both the overall early and late cure rates were $75 \%$. The use of energy sources other than the traditional cut-and-sew technique to create atrial lesions may be one reason for the variety of results, like the modifications of the maze procedure. ${ }^{5,6} \mathrm{It}$ seems that the cure rate after PVI has not yet reached the level achieved after Cox maze III. In our series, however, both the early and late cure rates in patients with normal SN function were almost $90 \%$, being comparable with the Cox maze procedure. If limited to patients with normal SN function, PVI appears to be satisfactorily effective for persistent/permanent AF. In patients without a corroboration of normal SN function, however, another procedure such as Cox maze III may be better than PVI.

\section{SN Function and Persistent/Permanent AF}

It is widely known that $\mathrm{SN}$ dysfunction is commonly associated with AF and that patients with SSS frequently have AF. ${ }^{10,11} \mathrm{SN}$ dysfunction has been attributed to fibrosis in the $\mathrm{SN}$ and atria, with slowed conduction throughout the atrial myocardium. In the presence of AF, further remodeling of the $\mathrm{SN}$ and the atrial myocardium can occur. ${ }^{10,14}$ It is 
thought that such histopathologic changes may be more severe in patients with a longer history of AF. In fact, a long $\mathrm{AF}$ duration is a significant risk factor for failure of surgical PVI and the Cox maze procedure in patients with persistent/permanent $\mathrm{AF}^{4,15}$

In our series, patients with $\mathrm{SN}$ dysfunction had a significantly lower $\mathrm{f}$ wave voltage in $\mathrm{V}_{1}$ of the ECG and a larger cardiothoracic ratio on chest $\mathrm{X}$-ray films than patients with normal SN function preoperatively. These findings suggest that patients with $\mathrm{SN}$ dysfunction may have a longer history of AF than patients with normal SN function. However, among patients with normal SN function, 1 patient who had the largest cardiothoracic ratio $(69 \%)$ in this series and a left atrial dimension of $67 \mathrm{~mm}$ on echocardiography preoperatively, maintained sinus rhythm throughout the follow-up period. It is still unclear which preoperative parameter is associated with $\mathrm{SN}$ dysfunction.

So far, there has been little information about the correlation between SN dysfunction and outcome after PVI. In the present study, we demonstrated that $\mathrm{SN}$ function can be safely assessed intraoperatively and that $27 \%(18 / 66)$ of the surgical candidates for persistent/permanent AF had pre-existing SN dysfunction. In such patients, both the early and late cure rates were significantly poorer than in patients with normal SN function. Thus the effect of PVI may be limited.

On the other hand, Hocini and coworkers ${ }^{16}$ have reported an improvement of SN recovery time after catheter-based PVI in patients with SSS. Khaykin and colleagues ${ }^{17}$ reported a 1-year follow-up after catheter-based PVI in patients with SSS, in which the occurrence of syncopal/ presyncopal events or documented sinus pause was significantly reduced after successful PVI in patients without permanent pacemakers $(n=12)$, and the percentage of atrial pacing was significantly reduced in patients with pacemakers $(\mathrm{n}=19)$. These findings suggest that histopathologic change in the SN and atria can be reversibly remodeled and that PVI can lead to improvement of SN function, although the mechanism responsible for the clinical improvement is still unknown. Thus PVI still seems to be an appropriate choice of therapy for some patients with $\mathrm{AF}$ who have $\mathrm{SN}$ dysfunction.

\section{Assessment of SN Function}

In our present series, intraoperative assessment of SN function was indicated for 66 of 76 patients with persistent/permanent AF. It is essential to confirm whether thrombus is present in the heart before cardioversion. The use of transesophageal echocardiography and complete unloading of the heart under extracorporeal circulation can minimize the risk of thromboembolism and make the patient more comfortable. From these viewpoints, intraoperative assessment is more advantageous than when the patient is awake. The 2 early nonfatal cerebral infarctions in our series did not seem to be associated with cardioversion because both occurred outside the perioperative period.

Among the 44 survivors in the normal SN function group, in whom the heart was defibrillated by cardioversion, $88 \%$ maintained their sinus rhythm during the follow-up period. In contrast, only $1(14 \%)$ patient maintained sinus rhythm among the 7 survivors in the SN dysfunction group, in whom the heart was not defibrillated. This suggests that response to cardioversion may be a simpler parameter for prediction of SN function. However, SN function is difficult to examine if a patient has a thrombus. The ideal method is one that can be done safely and quickly, and which is feasible for all patients.

\section{Limitations}

This study had certain limitations. First, it was a retrospective analysis, and the number of patients was relatively small. We acknowledge that large multicenter randomized prospective trials would provide the most reliable data for effective lesion sets in patients with persistent/permanent $\mathrm{AF}$ and $\mathrm{SN}$ dysfunction. However, it has been difficult to identify patients with SN dysfunction among those with persistent/permanent AF who are candidates for surgery. The merit of the present series was that it was a singlecenter study of operations performed by a single surgeon, with the longest follow-up period yet reported. Second, the present study lacks Holter ECG recordings. Finally, SN function was not completely examined in patients who could not be cardioverted intraoperatively. It should be noted that the $\mathrm{SN}$ dysfunction group in this series indicates patients without a corroboration of normal SN function.

\section{CONCLUSIONS}

Our follow-up study covering a period of up to 6 years after cut-and-sew PVI has demonstrated that the early and late cure rates of $\mathrm{AF}$ in patients with normal $\mathrm{SN}$ function were satisfactory and comparable with those after the Cox maze III procedure. In contrast, the results in patients with SN dysfunction were significantly poorer. Assessment of SN function may help to improve the success rate after PVI in patients with persistent/permanent AF associated with heart valve disease.

\section{References}

1. Kalil RAK, Lima GG, Leiria TLL, Abrahao R, Pires LM, Prates PR, et al. Simple surgical isolation of pulmonary veins for treating secondary atrial fibrillation in mitral valve disease. Ann Thorac Surg. 2002;73:1169-73.

2. Sueda T, Imai K, Orihashi K, Okada K, Ban K, Hamamoto M. Midterm results of pulmonary vein isolation for the elimination of chronic atrial fibrillation. Ann Thorac Surg. 2005;79:521-5.

3. Melby SJ, Zierer A, Bailey MS, Cox JL, Lawton JS, Munfakh N, et al. A new era in the surgical treatment of atrial fibrillation: the impact of ablation technology and lesion set on procedural efficacy. Ann Surg. 2006;244:583-92.

4. Gillinov AM, McCarthy PM, Blackstone EH, Rajeswaran J, Pettersson G, Sabik JF III, et al. Surgical ablation of atrial fibrillation with bipolar radiofrequency as the primary modality. J Thorac Cardiovasc Surg. 2005;129:1322-9. 
5. Gillinov AM, Bakaeen F, McCarthy PM, Blackstone EH, Rajeswaran J, Pettersson G, et al. Surgery for paroxysmal atrial fibrillation in the setting of mitral valve disease: a role for pulmonary vein isolation? Ann Thorac Surg. 2006; 81:19-28.

6. Stulak JM, Dearani JA, Sundt TM III, Daly RC, McGregor CGA, Zehr KJ, et al. Superiority of cut-and-sew techinique for the Cox maze procedure: comparison with radiofrequency ablation. J Thorac Cardiovasc Surg. 2007;133: 1023-7.

7. Cox JL, Boineau JP, Schuessler RB. Modification of the maze procedure for atrial flutter and atrial fibrillation. I. Rational and surgical results. J Thorac Cardiovasc Surg. 1995;110:473-83.

8. Cox JL. Evolving applications of the maze procedure for atrial fibrillation. Ann Thorac Surg. 1993;55:578-80.

9. Cox JL, Boineau JP, Schuessler RB, Boineau JP. Modification of the maze procedure for atrial flutter and atrial fibrillation. II. Surgical technique of the maze III procedure. J Thorac Cardiovasc Surg. 1995;110:485-95.

10. Kaplan BM, Langendorf R, Lev M, Pick A. Tachycardia-bradycardia syndrome (so-called "sick sinus syndrome"): pathology, mechanism and treatment. Am J Cardiol. 1973;31:497-508.
11. Rubenstein JJ, Schulman CL, Yurchak PM, DeSanctis RW. Clinical spectrum of the sick sinus syndrome. Circulation. 1972;46:5-13.

12. Mandel W, Hayakawa H, Danzig R, Marcus HS. Evaluation of sino-atrial node function in man by overdrive suppression. Circulation. 1971;44:59-66.

13. Shemin RJ, Cox JL, Gillinov AM, Blackstone EH, Bridges CR. Guidelines for reporting data and outcomes for the surgical treatment of atrial fibrillation. Ann Thorac Surg. 2007;83:1225-30.

14. Thery C, Gosselin B, Lekieffre J, Warembourg H. Pathology of sinoatrial node. Correlations with electrocardiographic findings in 111 patients. Am Heart J. 1997;93:735-40.

15. Itoh A, Kobayashi J, Bando K, Niwaya K, Tagusari O, Nakajima H, et al. The impact of mitral valve surgery combined with maze procedure. Eur J Cardiothorac Surg. 2006;29:1030-5.

16. Hocini M, Sanders P, Deisenhofer I, Jais P, Hsu LF, Scavee C, et al. Reverse remodeling of sinus node function after catheter ablation of atrial fibrillation in patients with prolonged sinus pauses. Circulation. 2003;108:1172-5.

17. Khaykin Y, Marroghche N, Martin DO, Saloba W, Schweikert R, Wexman M, et al. Pulmonary vein isolation for atrial fibrillation in patients with symptomatic sinus bradycardia or pauses. J Cardiovasc Electrophysiol. 2004;15:784-9. 